\section{Single cell and spatial transcriptomics in human tendon disease indicate dysregulated immune homeostasis}

Tendinopathy; encompassing multifactorial tendon disorders characterised by pain and functional limitation remains a significant burden in musculoskeletal medicine. ${ }^{1}$ Recent findings highlight a key role for immune mediated mechanisms in tendon disease supporting the concept that pivotal immunological and biomechanical factors conventionally associated with inflammatory rheumatic and musculoskeletal diseases (RMDs) are manifest in tendon. ${ }^{2}$ Single cell technologies ${ }^{3}$ (scRNAseq) are increasingly applied in rheumatology to identify key cellular phenotypes that drive disease pathogenesis. Despite efforts with small cell numbers and heterogenous tendon biopsies ${ }^{4}$ there remains no detailed spatial tendon cell atlas to inform translational targeting. Herein, for the first time utilising scRNAseq and spatial transcriptomics $\left(\mathrm{S}_{\mathrm{T}}\right)$, we carry out cell-cell interaction analysis to build an atlas of the dynamic cellular environment that drives the development of chronic human tendon disease.

In healthy ( 4 biopsies, $n=3040$ cells) and diseased ( 5 biopsies, $\mathrm{n}=19084$ cells) tendon we find a mix of endothelial, immune and stromal cells (figure 1A, online supplemental file 1). Each cell type group is present in disease and healthy tissue but with distinct quantitative and qualitative characteristics. Within stromal populations we identified 'mural type' stromal cells (figure 1B). Mural cells, which include pericytes, are possible progenitor cells in tendon ${ }^{5}$ and interestingly, these cells are phenotypically similar to NOTCH3 high mural cells described in rheumatoid arthritis (RA) synovium which can differentiate into fibroblasts following interactions with endothelial cells (ECs) via JAG1. ${ }^{6}$ Cell-cell interaction and $\mathrm{S}_{\mathrm{T}}$ analysis indicate a similar phenomenon could occur within tendinopathy between mural cells and SEMA3G ECs (figure 1F). In all diseased stromal cell populations, there was greater expression of genes for extracellular matrix proteins (eg, COL1A1, COL3A1, FN1, $B G N$ ) which is considered the hallmark feature of tendinopathy (online supplemental figure S2B). Furthermore, pathway analysis indicates stromal cell clusters shift from negative regulation of immune cell and cytokine responses in normal tendon (online supplemental figure S3A) to a state that promotes immune cell recruitment and activation along with cytokine secretion and response processes in diseased tendon (online supplemental figure S3B,C).

Seven subtypes of PECAM1 + ECs were found (figure 1D), including a population of LYVE1+ ECs that produce CCL21 and have been shown to regulate dendritic cell (DC) migration. ${ }^{7}$ Furthermore, CCL21 is upregulated in these cells in tendinopathy (online supplemental figure S2C). DCs comprise the single largest immune cell population present in normal tendon (figure 1B). Intriguingly, DCs are also present in diseased tendon however, showing therein greater levels of DC activation and lower levels of C1Q genes (regulatory DC markers) (online supplemental figure S2D,E). The activation of DCs and subsequent $\mathrm{T}$ cell activation ${ }^{8}$ in tendinopathy is further evidenced by pathway analysis of differentially upregulated genes in disease (online supplemental figure S3D,E). This activation may in part be due to increased matrix protein expression, such as FN1, which can activate DCs and resulting in alterations in T cell populations within tissue potentially contributing to mechanisms driving disease chronicity. Additionally, we found three populations of macrophages in diseased tendon, one of which, cycling macrophages (figure $1 \mathrm{C}$, online supplemental figure S2A) is unique to diseased tissue. The transcript profile of macrophages in normal tendon most closely resembled tissue repair and debris clearance (figure 1E, online supplemental figure S2D). Within normal tendon we found APP expression in tenocytes which can induce a resolution promoting phenotype in macrophages. However, $A P P$ expression was reduced in the stromal compartment in disease coinciding with diminished expression of LYVE1 within diseased tissue macrophages (figure 1E, online supplemental figure S2D). These macrophage subsets have recently been associated with RA remission and we postulate the phenotypic drift away from this phenotype promotes aberrant tissue repair and attendant tendinopathy. ${ }^{9}$

Further evidence suggesting that the stromal environment may induce inflammatory changes comprises increased expression of MIF (figure 1E, online supplemental figure S2B) in diseased tenocytes that can induce proinflammatory effects via its receptor $C D 74$, which is also upregulated in macrophages from diseased tendon (figure $1 \mathrm{E}$, online supplemental figure $\mathrm{S} 2 \mathrm{C}) . \mathrm{S}_{\mathrm{T}}$ generated indicative data from cell-cell interaction analysis suggests stromal induced immune regulation. As such, we postulate the primary role of the immune compartment within the tendon is to regulate and resolve damage; however, following cumulative microtrauma the fundamental process of debris removal and matrix repair initiated by tenocytes could lead to positive amplification of the immune compartment. We further propose that within diseased tendon immune homoeostasis may become imbalanced and activated immune cells, primed by both endothelial and stromal cells, promote a cycle of inflammation and aberrant tissue repair. The inflammatory environment, including cytokine pathways that are unequivocally demonstrated in this preliminary tendon atlas, have been targeted to yield potent immunological interventions in a range of inflammatory RMDs-the potential to target and investigate these pathways in human tendon disease is now compelling.

Moeed Akbar $\odot,{ }^{1}$ Lucy MacDonald $\odot,{ }^{1,2}$ Lindsay A N Crowe, ${ }^{1}$ Konstantin Carlberg $\odot{ }^{3}$ Mariola Kurowska-Stolarska $\odot 1,2$ Patrik L Ståhl, ${ }^{3}$ Sarah J B Snelling, ${ }^{4}$ lain B Mclnnes, ${ }^{1,2}$ Neal L Millar ${ }^{1}$

${ }^{1}$ Institute of Infection, Immunity and Inflammation, College of Medical Veterinary and Life Sciences, University of Glasgow, Glasgow, UK

${ }^{2}$ Research into Inflammatory Arthritis Centre Versus Arthritis (RACE), University of Glasgow, Glasgow, UK

${ }^{3}$ Science for Life Laboratory, Dept. of Gene Technology, KTH Royal Institute of Technology, Solna, Sweden

${ }^{4}$ Nuffield Department of Orthopaedics, Rheumatology and Musculoskeletal Sciences, University of Oxford, Oxford, UK

Correspondence to Mr Neal L Millar, Institute of Infection, Immunity and Inflammation, University of Glasgow, Glasgow G12 8TA, UK; neal.millar@glasgow.ac.uk

Handling editor Josef S Smolen

Twitter Neal L Millar @tendonglasgow

Acknowledgements We thank the CZI Tendon Seed Network for helpful discussions when preparing this manuscript for publication.

Contributors MA, LM and NM conceived and designed the experiments. MA, LM and KC performed experiments. IBM, NM, LANC, PS and SJBS provided expert advice. All authors analysed the data. MA, LANC, IBM and NM wrote the paper.

Funding This work was funded by the Medical Research Council (MR/R020515/1). SJBS was funded by the NIHR Oxford Biomedical Research Centre

Competing interests $\mathrm{KC}$ and PS are scientific consultants to 10x Genomics.

Patient consent for publication Not required.

Ethics approval All procedures and protocols were approved by the Ethics Committee under the approval number West of Scotland REC (REC14/WS/1035) with 

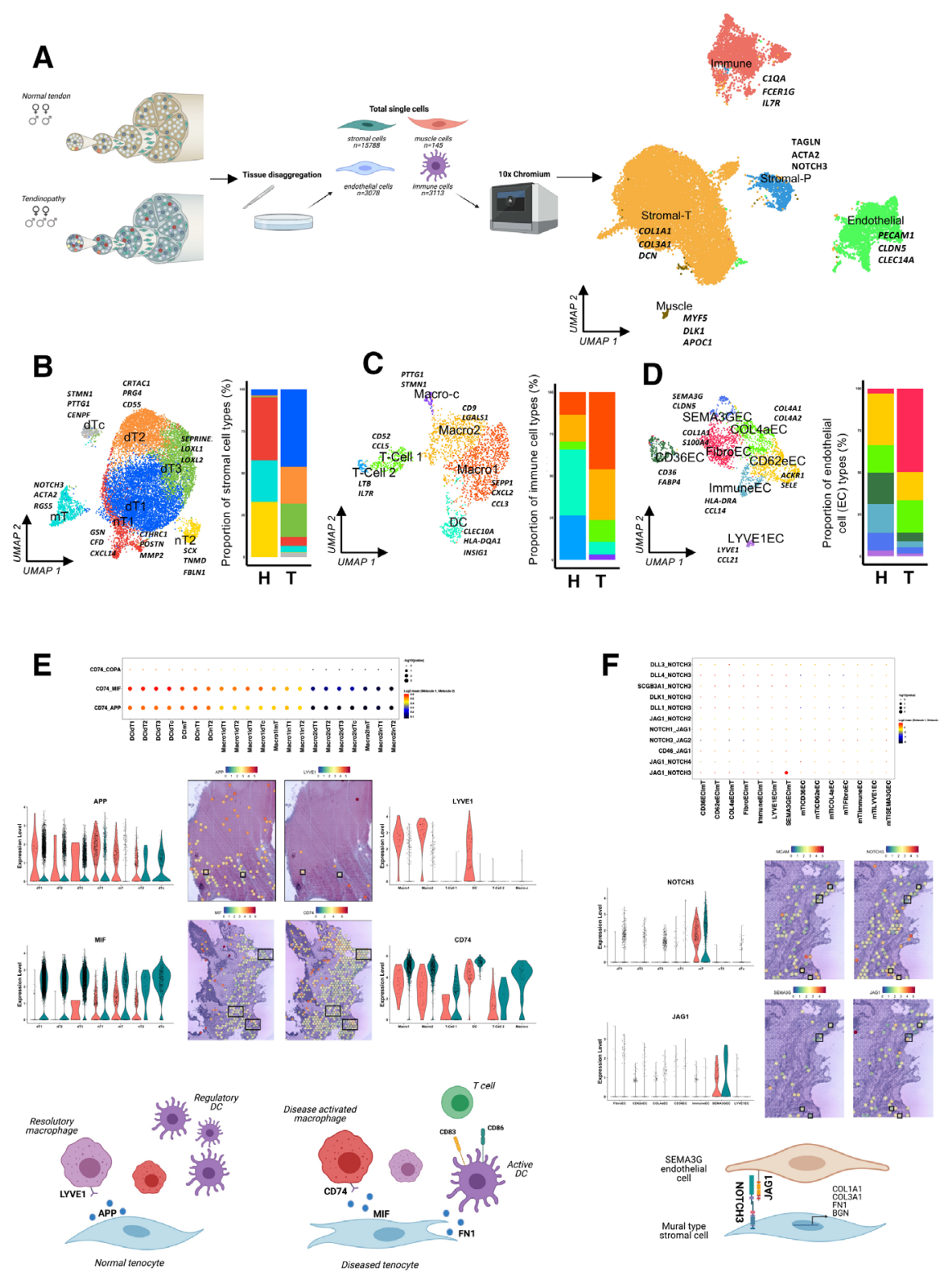

Figure 1 Cell composition and interactions of healthy and diseased human tendon. (A) Normal ( $n=4$, human hamstring tendon) and diseased (tendinopathy, $\mathrm{n}=5$, human supraspinatus tendon) human tendon were processed for single cell analysis using Chromium 10x 3'DEG chemistry. Infographic shows number of donors and cells sequenced. Uniform manifold approximation and projection (UMAP) embedding of 22124 single cells delineating endothelial, immune, stromal tenocyte and stromal mural cells with marker genes. (B) Stromal cells of the tendon. UMAP embedding with gene markers and distribution of seven delineated stromal cell populations from human tendons; mural tenocyte (mT), normal tenocyte1 (nT1), normal tenocyte2 (nT2), diseased tenocyte1 (dT1), diseased tenocyte2 (dT2), diseased tenocyte3 (dT3) and diseased cycling tenocytes (dTc). (C) Immune cells of the tendon. UMAP embedding with gene markers and distribution of 6 delineated immune cell populations from human tendons; dendritic cells (DC), macrophage1 (Macro1), macrophage1 (Macro2), cycling macrophage (Macro-C), T-Cells1 (T-Cell1) and T-Cells2 (T-Cell2). (D) Endothelial cells $(E C)$ of the tendon. UMAP embedding with gene markers and distribution of seven delineated EC populations from human tendons; CD36 high EC (CD36EC), E-Selectin EC (CD62eEC), collagen 4 vessel EC (COL4aEC), immune-like EC (ImmuneEC), LYVE1 positive EC (LYVE1EC) and SEMA3G positive EC (SEMA3GEC). (E) Tenocyte-immune interactions in tendon ( $\mathrm{n}=3$ healthy, vs 4 diseased). Predicted cell-cell interactions using CellphoneDB statistical framework on human tendon immune and stromal cells. Selected ligand receptor interactions showing APP and MIF ligandreceptor pairs in tendon stromal and immune cells. Mean of combined gene expression of interaction pairs (Log2 mean) and p value of specificity of interactions. Violin plots of APP and MIF expression in tendon stromal cells from healthy (pink) and diseased (green) tendon. Spatial expression (log2FC) of stromal APP and macrophage LYVE1 in normal human tendon and stromal MIF and macrophage CD74 in tendinopathic tendon visualised on 10x Genomics visium data, boxes highlight areas of coexpression. Violin plots of LYVE1 and CD74 expression in immune cells from healthy (pink) and diseased (green) tendon. Biorender infographic summarising tenocyte-immune cell interactions in tendon disease. (F) EC-tenocyte interactions in tendon. Predicted cell-cell interactions using CellphoneDB statistical framework on selected human tendon endothelial and stromal cells. Ligand-receptor interactions showing NOTCH3 ligand-receptor pairs in tendon endothelial and stromal cells. Mean of combined gene expression of interaction pairs (Log2 mean) and p value of specificity of interactions. Violin plots of NOTCH3 and JAG1 expression in tendon stromal and ECs, respectively from healthy (pink) and diseased (green) tendon. Spatial expression (log2FC) of NOTCH3 and MCAM from mural tenocytes and SEMA3G and JAG1 from SEMA3GEC's in human diseased tendon visualised on 10x Genomics visium data, boxes highlight areas of coexpression. Biorender graphic of predicted SEMA3GEC and $\mathrm{mT}$ interaction in human tendon. 
informed consent obtained and carried out in accordance with standard operative procedures

Provenance and peer review Not commissioned; externally peer reviewed.

Supplemental material This content has been supplied by the author(s). It has not been vetted by BMJ Publishing Group Limited (BMJ) and may not have been peer-reviewed. Any opinions or recommendations discussed are solely those of the author(s) and are not endorsed by BMJ. BMJ disclaims all liability and responsibility arising from any reliance placed on the content. Where the content includes any translated material, BMJ does not warrant the accuracy and reliability of the translations (including but not limited to local regulations, clinical guidelines, terminology, drug names and drug dosages), and is not responsible for any error and/or omissions arising from translation and adaptation or otherwise.$$
\text { (2) }
$$$$
\text { OPEN ACCESS }
$$

Open access This is an open access article distributed in accordance with the Creative Commons Attribution 4.0 Unported (CC BY 4.0) license, which permits others to copy, redistribute, remix, transform and build upon this work for any purpose, provided the original work is properly cited, a link to the licence is given, and indication of whether changes were made. See: https://creativecommons.org/ licenses/by/4.0/.

(c) Author(s) (or their employer(s)) 2021. Re-use permitted under CC BY. Published by BMJ.

- Additional supplemental material is published online only. To view, please visit the journal online (http://dx.doi.org/10.1136/annrheumdis-2021-220256).

\section{A) Check for updates}

To cite Akbar M, MacDonald L, Crowe LAN, et al. Ann Rheum Dis Epub ahead of print: [please include Day Month Year]. doi:10.1136/annrheumdis-2021-220256
Received 1 March 2021

Accepted 5 May 2021

Ann Rheum Dis 2021;0:1-3. doi:10.1136/annrheumdis-2021-220256

ORCID iDs

Moeed Akbar http://orcid.org/0000-0002-6923-4724

Lucy MacDonald http://orcid.org/0000-0001-7727-3873

Konstantin Carlberg http://orcid.org/0000-0002-1774-6058

Mariola Kurowska-Stolarska http://orcid.org/0000-0003-2124-7777

Neal L Millar http://orcid.org/0000-0001-9251-9907

\section{REFERENCES}

1 Millar NL, Silbernagel KG, Thorborg K, et al. Tendinopathy. Nat Rev Dis Primers 2021;7:1.

2 Gracey E, Burssens A, Cambré I, et al. Tendon and ligament mechanical loading in the pathogenesis of inflammatory arthritis. Nat Rev Rheumatol 2020;16:193-207.

3 Cheung P, Khatri P, Utz PJ, et al. Single-cell technologies - studying rheumatic diseases one cell at a time. Nat Rev Rheumatol 2019:15:340-54.

4 Kendal AR, Layton T, Al-Mossawi H, et al. Multi-Omic single cell analysis resolves novel stromal cell populations in healthy and diseased human tendon. Sci Rep 2020;10:13939.

5 De Micheli AJ, Swanson JB, Disser NP, et al. Single-Cell transcriptomic analysis identifies extensive heterogeneity in the cellular composition of mouse Achilles tendons. Am J Physiol Cell Physiol 2020;319:C885-94.

6 Wei K, Korsunsky I, Marshall JL, et al. Notch signalling drives synovial fibroblast identity and arthritis pathology. Nature 2020;582:259-64.

7 Murphy PM. Double duty for CCL21 in dendritic cell trafficking. Immunity 2010:32:590-2.

8 Garcia-Melchor E, Cafaro G, MacDonald L, et al. Novel self-amplificatory loop between $T$ cells and tenocytes as a driver of chronicity in tendon disease. Ann Rheum Dis 2021. doi:10.1136/annrheumdis-2020-219335. [Epub ahead of print: 10 Mar 2021] (published Online First: 2021/03/12).

9 Alivernini S, MacDonald L, Elmesmari A, et al. Distinct synovial tissue macrophage subsets regulate inflammation and remission in rheumatoid arthritis. Nat Med 2020;26:1295-306. 